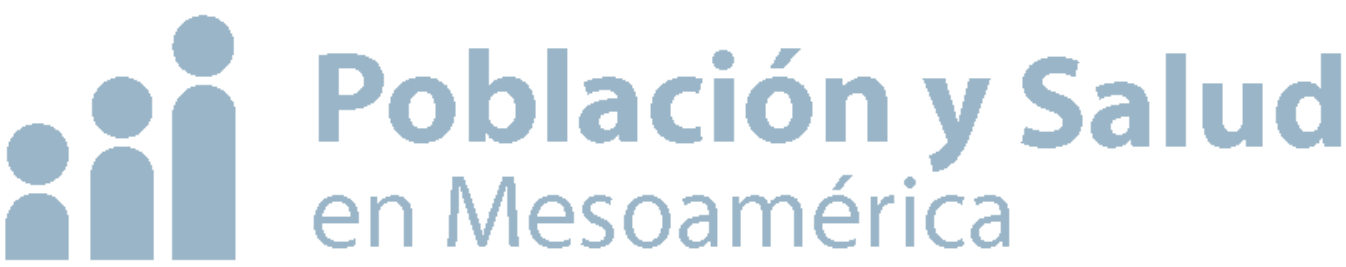

\section{Factores asociados al incremento de cesáreas en México, 2011-2014}

José Alberto Muños Hernández, María Graciela Freyermuth Enciso y María del Pilar Ochoa Torres

\section{Como citar este artículo:}

Muños Hernández, J. A., Freyermuth Enciso, M. G. y Ochoa Torres, M. (2020) Factores asociados al incremento de cesáreas en México, 2011-2014. Población y Salud en Mesoamérica, 17(2). Doi: https://doi.org/10.15517/psm.v17i2.40046

\section{@(1) (2)}

ISSN-1659-0201 http://ccp.ucr.ac.cr/revista/

Revista electrónica semestral

Centro Centroamericano de Población Universidad de Costa Rica 
Factores asociados al incremento de cesáreas en México, 2011-2014

Factors associated with the increase in cesareans in Mexico, 2011-2014 José Alberto Muños Hernández ${ }^{1}$ María Graciela Freyermuth Enciso² María Pilar Ochoa Torres ${ }^{3}$

Resumen: Introducción: analizamos la prevalencia del parto vía cesárea en México para 2011-2014, buscando identificar algunos de los factores asociados a la presencia de cesárea durante el parto. Metodología: se realizó un análisis descriptivo y un modelo logístico multinivel con los registros de nacimientos del periodo 2011-2014, que incluye las características de la madre y del contexto. Resultados: se identificó que la presencia de factores relacionados con una mayor posibilidad de que el parto resulte en cesárea son mujeres que habitan municipios con población indígena, mayor escolaridad, mayor número de consultas prenatales, inicio temprano de estas y atenderse en clínicas privadas. También se identificó una mayor posibilidad de estancias prolongadas de hospitalización del parto si es por cesárea. Conclusiones: se observa un incremento en el número de cesáreas en hospitales públicos a partir del esquema de aseguramiento del Seguro Popular y se confirma la relación directa entre los múltiples factores analizados y la cesárea, entre ellos, mayor escolaridad, habitar en municipios predominantemente indígenas, así como en municipios con mayor índice de desarrollo humano.

Palabras clave: Cesárea, medicalización, parto, violencia, derechos reproductivos, México.

Abstract: Introduction: The objective is to analyze the prevalence of delivery by cesarean section in Mexico among 2011-2014, to identify some of the factors associated with its occurrence. Methods: A descriptive analysis and a multilevel logistic model was conducted among the data for Birth Information Subsystem, characteristics for the mother and context. Results: Identified that the factors associated with a greater likelihood that delivery result in cesarean section, are higher levels of education, greater number of prenatal visits, an early start to prenatal visits, giving birth in private health. Also a greater likelihood of prolonged hospitalization when delivery is by cesarean section. Conclusions: An increase in the number of caesarean sections in public hospitals is observed from the Popular Insurance assurance scheme; and the direct relationship between the multiple factors analyzed and the caesarean section is confirmed, including higher education, living in predominantly indigenous municipalities, as well as in municipalities with the highest human development index.

Key words: Caesarean section, medicalization, birth, violence, reproductive rights, Mexico.

Recibido: 08 Jul 2019 | Corregido: 23 nov 2019 | Aceptado: 01 Oct 2019

\footnotetext{
${ }^{1}$ Centro de Investigaci ones y Estudios Superiores en Antropología Social (CIESAS), MÉXICO. MÉXICO.jmunos@colmex.mx ; kasteblok@yahoo.com.mx. ORCID: https://orcid.org/0000-0001-6128-8579

${ }^{2}$ Centro de Investigaciones y Estudios Superiores en Antropología Social (CIESAS)-Unidad Sureste y Secretaria Técnica del Observatorio de Mortalidad Materna en México (OMM). MÉXICO. gracielafreyermuth54@hotmail.com.ORCID: https://orcid.org/0000-0002-3871-715X

${ }_{3}^{3}$ Subsecretaría de Integración y Desarrollo del Sector Salud. MÉXICO. pilarochoatorres@gmail.com. ORCID: https://orcid.org/0000-0002-0791-1806
} 


\section{Introducción}

En 1922, J. Whitridge Williams afirmaba que "la excelencia de un obstetra se debe estimar no por el número de cesáreas que realiza, sino más bien por las que no realiza"4

Desde la década los ochenta los profesionales de la salud han considerado que la tasa de cesáreas ${ }^{5}$ debe oscilar entre 10 y 15 \% (Betrán et al., 2015). ${ }^{6}$ Independientemente de la región de que se trate, se considera que no hay justificación para que sea mayor en vista del porcentaje esperado de complicaciones obstétricas. A pesar de esto, la cesárea es una de las cirugías más realizadas y su práctica es cada vez más frecuente, tanto en países desarrollados como en desarrollo (Organización Mundial de la Salud [OMS] y Human Reproductive Program, 2015). Esta práctica se ha vuelto también habitual en México, donde se ha observado un incremento importante en el porcentaje de cesáreas en la última década en todas las entidades federativas.

Este procedimiento ya se utilizaba en el siglo XVIII para el parto obstruido y empezó a realizarse con más frecuencia en el siglo XIX, aunque con tasas de Mortalidad Materna (MM) bastante elevadas (68 a 85 \%) (Gómez-Dantés, 2004). Luego de los adelantos tecnológicos entrado el siglo XIX, no solamente fue posible mejorar la técnica de la cesárea, sino también el control de las hemorragias, la asepsia y la antisepsia (Araujo, Encinas, Torres, y Caballero, 2011), los insumos, las técnicas en la anestesia (mediados del siglo XIX) (Becerro de Bengoa-Callau y Becerro de Bengoa-García, 2001; Celesia, 2004; Pinós, 1997), las transfusiones sanguíneas (Institute of Biomedical Science (IBMS, 2005); Sarkar, 2008; Young, 1964; Lane y Lamkin, 1984) y el uso de antibióticos y analgésicos (Becerro de Bengoa y Becerro de Bengoa, 2001; Belloso, 2009). Durante la primera mitad del siglo XX, la cesárea fue un procedimiento terapéutico para indicaciones muy precisas: placenta previa, desprendimiento prematuro de placenta, inducción fallida del trabajo de parto, cesárea previa, preeclampsia severa/eclampsia, prolapso del cordón umbilical, sufrimiento fetal, presentación incompleta de nalgas/presentación anómala, desproporción cefalopélvica/ trabajo de parto prolongado, gestación múltiple, fístula vesicovaginal, y, más recientemente, presencia de enfermedades virales en la madre (Virus de Inmunodeficiencia Humano -VIH-, Virus del Papiloma Humano). A mediados del siglo XX, este procedimiento se incorporó definitivamente a las técnicas

4 Ronald M. Cyr, "Myth of the ideal cesarean section rate: Commentary and historic perspective", AJOG, (2006) 194; 932-6.

5 Porcentaje de partos por cesárea entre el número total de nacidos vivos.

6 Conclusiones de la Reunión de Profesionales de la Salud realizada en Brasil en 1985, convocada por la Organización Mundial de la Salud: http://apps.who.int/iris/bitstream/10665/161444/1/WHO_RHR_15.02_spa.pdf?ua=1. 
de la obstetricia, aunque posteriormente se salió de control hasta llegar a una desmesura injustificada (Gómez-Dantés, 2004).

Entre los elementos que han contribuido al incremento de la cesárea se encuentra el desarrollo de los instrumentos para el monitoreo fetal, que elevó enormemente este procedimiento por los trazos "desalentadores" observados por las personas obstetras. El sufrimiento fetal, distocia, presentación anormal y cesárea previa han constituido 90 \% de las indicaciones (Secretaría de Salud [SSA], 2009a), argumentándose a favor de su incremento que es un procedimiento con una morbilidad y mortalidad materna y neonatal relativamente bajas. Sin embargo, no está demostrado que este procedimiento traiga consigo beneficios para la mujer o el nonato cuando es innecesario (Belloso, 2009). Incluso algunos autores han identificado una asociación positiva entre el tratamiento antibiótico poscesárea y la mortalidad y morbilidad materna severa y una relación entre el incremento de las tasas de cesárea y el incremento en las tasas de muerte fetal (Villar et al, 2006).

En la literatura internacional se han documentado distintas razones por las que el personal médico decide realizar un parto por cesárea y no por vía vaginal. Entre estas destaca el que la cesárea resulta más conveniente para los médicos, ya que les permite el control del lugar y la fecha en que ocurre un parto. Esto facilita que puedan programar el nacimiento de acuerdo con sus prioridades de horario y sus compromisos profesionales o personales (Graham et al, 1999). Otra razón es que los honorarios que perciben son más elevados y no invierten tiempo en el cuidado del trabajo de parto, por lo que el proceso del nacimiento puede durar una hora y con el cuidado posoperatorio una hora más. Así, en dos horas el parto se habrá resuelto. Incluso la posición corporal para la atención es más cómoda durante una operación cesárea, pues en la mesa de quirófano se mantiene el control total del cuerpo de la embarazada, que no se queja o grita, ni se mueve. El procedimiento suele ser más "fácil", ya que se cuenta con el auxilio de más personal que en un parto vaginal: cuatro personas por lo menos (Plante, 2006).

Adicional a que la atención de un parto vaginal requiere de mayor cuidado, su desarrollo puede llevar más de 12 horas y alrededor de una hora u hora y media más para el nacimiento y alumbramiento. En una sala de labor puede haber varias mujeres en el mismo trance, ya que no se tiene control sobre el momento del nacimiento del bebé. En salas de parto sobresaturadas este puede ser un problema para la atención. La Federación Mexicana de Colegios de Obstetricia y Ginecología (FEMECOG) ha manifestado -a través de un pronunciamiento en junio de 2015- que los médicos en las instituciones públicas de salud no están en posibilidades de dar acompañamiento a un trabajo de parto y nacimiento en condiciones deseables y que, por ello, se ha incrementado en nuestro país el número de cesáreas (FEMECOG, 2015). Cabe señalar que en las últimas décadas las políticas para disminuir la MM han estado encaminadas a la atención intrahospitalaria del parto, haciendo a un lado el primer nivel de atención y sobresaturando el nivel hospitalario con la atención de partos normales (Sesia, García, García, Carmona y Sachse, 2013). 
Un estudio realizado por Betrán et al (2007) indagó sobre la relación existente entre las tasas de cesárea y la razón de mortalidad materna a partir de un método de regresión no paramétrica. Para ello, los autores utilizaron datos provenientes de encuestas y registros administrativos de 129 países sobre nacimientos vía cesárea y encontraron que la tasa promedio estimada de cesáreas era de 15 \%. Al relacionar esta tasa propuesta con la razón de mortalidad materna (RMM) por país, aun cuando la tendencia pareciera mostrar que a mayor tasa de cesáreas menor $\mathrm{RMM}$, no se tiene una correlación significativa.

Para este estudio, los valores utilizados para cada país fueron los siguientes, para África, la tasa promedio de cesáreas fue de 3,5\%, con los valores más altos para Sudáfrica (15,4\%), Egipto $(11,4$ \%) y Túnez (8,0 \%), y RMM de 90, 78 y 43 respectivamente. En Asia, el promedio de cesáreas fue de $15,9 \%$, siendo China (40,5 \%), Hong Kong (27,4 \%) y Líbano (23,3 \%) los países con las tasas mayores, y RMM de 52 para China y 32 para Líbano (Gapminder, S.F.). En Nepal y Camboya se observaron las menores tasas de cesárea (1,0 \%) con RMM de 384 y 457, respectivamente, mientras que en Europa el promedio fue de $19 \%$, mostrando los valores más altos en Italia con $36 \%$ y Portugal con 30,2 \%, y RMM de 6,8 y 16, respectivamente (Gapminder, S.F.; Betrán et al, 2007).

En América Latina y el Caribe las tasas de cesáreas superaron a las de otros países en vías de desarrollo, pero no fueron más altas que las de los países desarrollados en promedio (Gapminder, S.F.). Según los autores de este reporte, los valores menores se observaron en Haití con 1,7 \% y en Honduras con 7,9 \% y RMM de 555 y 133, respectivamente (Betrán et al, 2007); la tasa media de cesáreas en esta región fue de 29,2 \% y las tasas más altas se presentaron en México (39,1 \%), Brasil (36,7 \%), República Dominicana (31,3\%) y Chile (30,7\%), con respectivas RMM de 66, 67, 62 y 23.

Otro aspecto que considerar es la relación entre el personal médico y las usuarias de los servicios o clientes (como algunos médicos privados las denominan). Esta es una relación totalmente asimétrica, más aún cuando se tiene que tomar una decisión sobre el tipo de procedimiento por el cual se va a atender el parto: vaginal o cesárea. Por un lado, el argumento de que el nonato está en peligro de sufrir algún daño pocas veces puede ser rebatido por una mujer embarazada y su familia $y$, por otro lado, hay ausencia de información sobre las complicaciones y riesgos en salud, para la madre y el producto, que pueden originarse mediante la operación cesárea y la anestesia. El monopolio del conocimiento en este campo lo tienen los obstetras, por lo que es difícil refutarlo inclusive para los médicos no obstetras (Gómez-Dantés, 2004).

Es importante señalar que este procedimiento bien utilizado contribuye a salvar vidas y que sus resultados dependen también de la calidad de la atención (Secretaría de Salud, 1995). Si el procedimiento se utilizara solamente bajo una perspectiva terapéutica, su distribución no presentaría grandes diferencias entre las mujeres mexicanas, pero no es así. A menudo los médicos 
tienen la percepción de que realizar una cesárea representa para la paciente, más beneficios que riesgos (Emmett, Shaw, Montgomery, Murphy y Diamond Study Group, 2006).

Según un análisis comparativo de la Encuesta Nacional de Salud y Nutrición (ENSANUT) de 2000, 2006 y 2012, en México la cesárea se ha incrementado 50 \% en 12 años, con un crecimiento diferenciado entre los sectores público y privado de la medicina, crecimiento que ha sido de $33 \%$ y 66\%, respectivamente (ENSANUT, 2012). A nivel nacional, entre 2011 y 2014 hubo 8746144 nacimientos, de los cuales casi la mitad (4 012 894) fueron por cesárea (Ver Figura 1). Así mismo, la relación entre cesáreas y disminución de la RMM en México no queda clara, ya que hay estados con 50 \% de cesáreas y RMM menores a la nacional, como Jalisco, Nuevo León, Sinaloa y Tlaxcala (datos para 2013), y entidades como Yucatán y Tamaulipas con altos porcentajes de cesáreas y RMM por arriba de la nacional. Por otro lado, se observa que hay estados como San Luis Potosí con la tasa más baja de cesáreas y una RMM menor a la media nacional, en cambio otros con porcentajes algo más reducidos de cesáreas y RMM elevadas, como Guerrero y Chiapas (Ver Figura 2).

\section{Figura 1}

Evolución de la distribución porcentual del parto por cesárea, México, 2011-2014

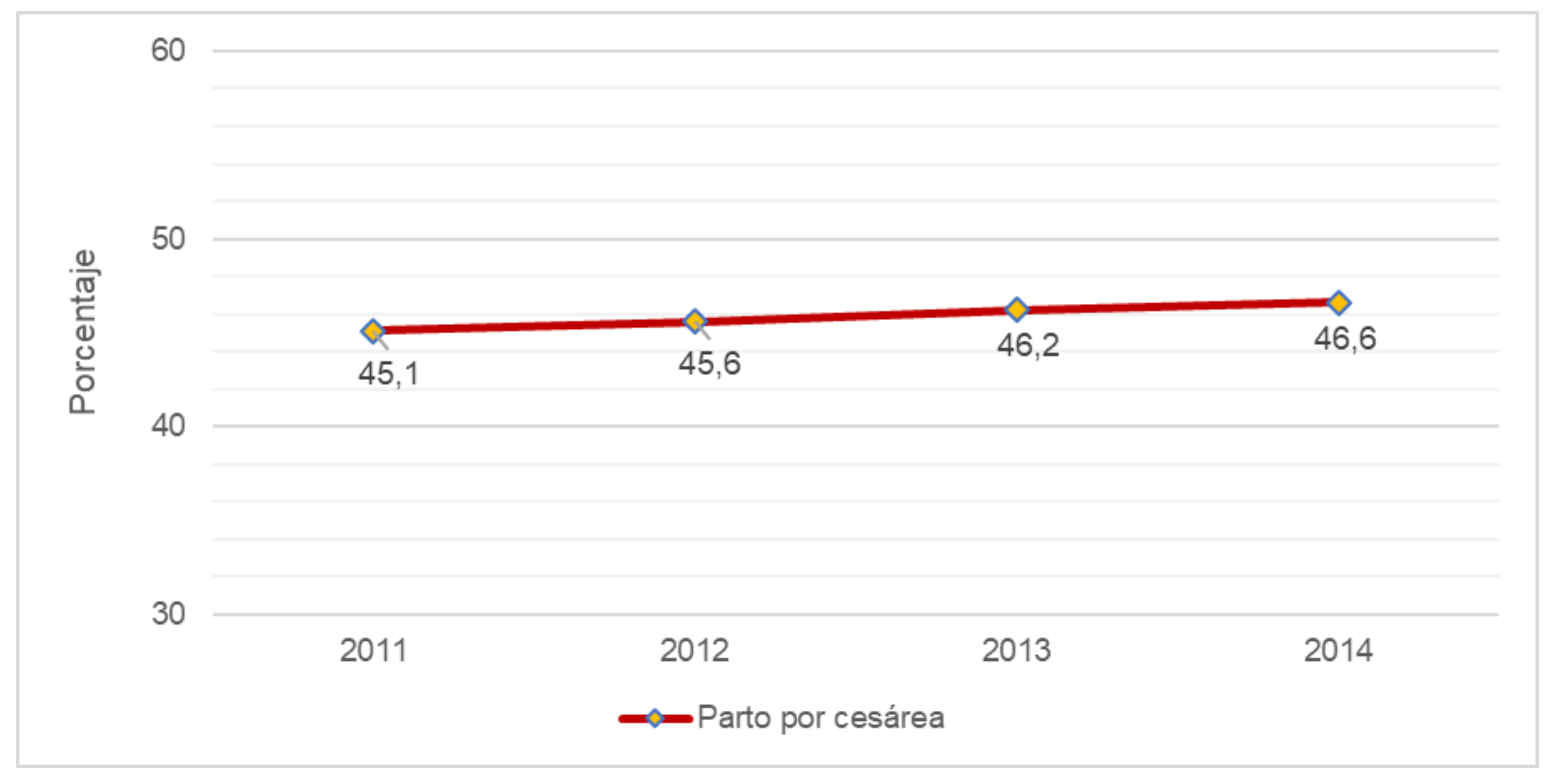

Fuente: Elaboración propia basada en información de la DGIS (2014), SINAC 2011-2014. 


\section{Figura 2}

Distribución porcentual de partos por cesárea, según entidad federativa, México, 2013

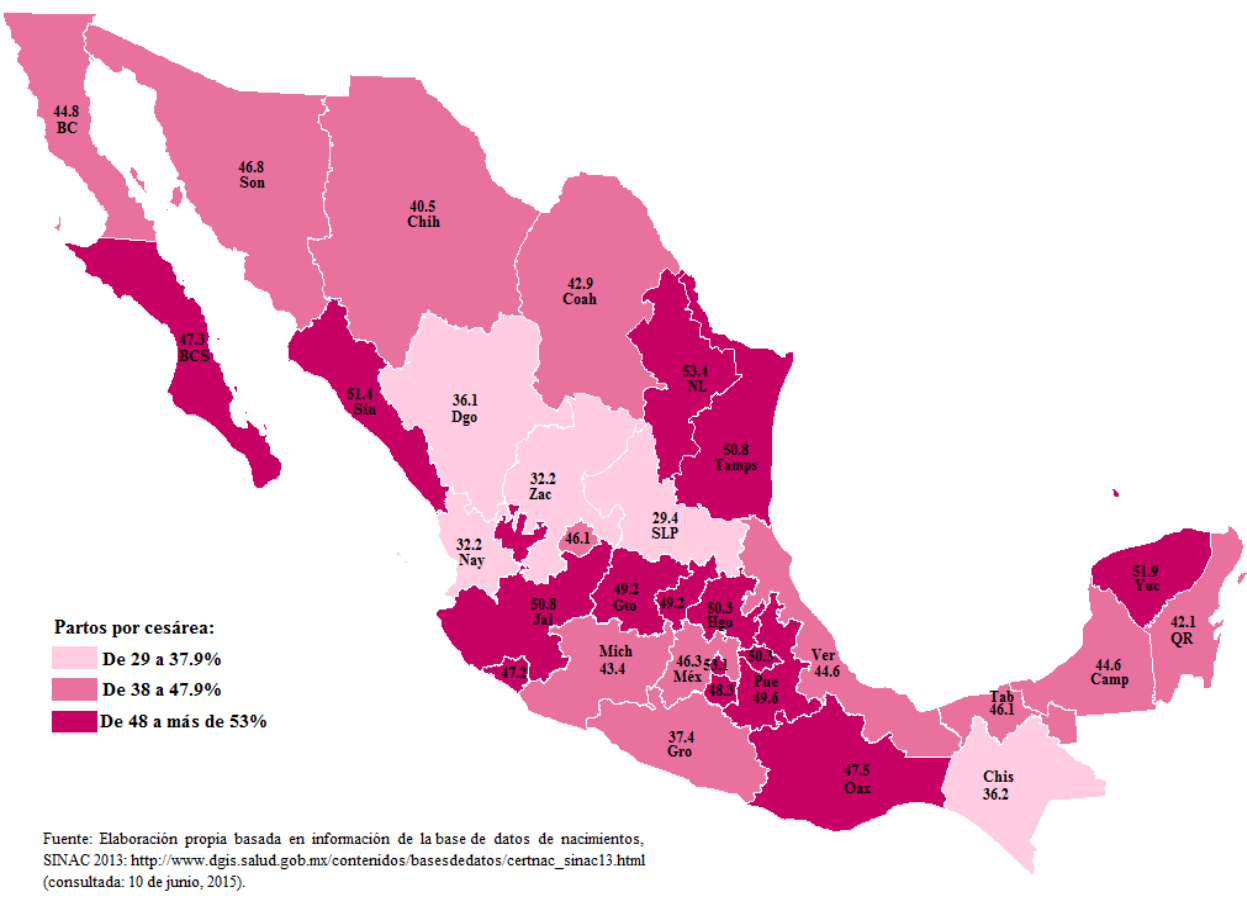

\begin{tabular}{lrr} 
Entidad & $\begin{array}{r}\text { Parto } \\
\text { vaginal }\end{array}$ & Cesárea \\
\hline Nacional & $\mathbf{5 2 . 7}$ & $\mathbf{4 6 . 2}$ \\
Aguascalientes & $\mathbf{5 2 . 9}$ & $\mathbf{4 6 . 1}$ \\
Baja California & $\mathbf{5 4 . 8}$ & $\mathbf{4 4 . 8}$ \\
Baja California Sur & $\mathbf{5 2 . 1}$ & $\mathbf{4 7 . 3}$ \\
Campeche & $\mathbf{5 5 . 3}$ & $\mathbf{4 4 . 6}$ \\
Chiapas & $\mathbf{6 3 . 5}$ & $\mathbf{3 6 . 2}$ \\
Chihuahua & $\mathbf{5 8 . 8}$ & $\mathbf{4 0 . 5}$ \\
Coahuila & $\mathbf{5 5 . 7}$ & $\mathbf{4 2 . 9}$ \\
Colima & $\mathbf{5 2 . 6}$ & $\mathbf{4 7 . 2}$ \\
Distrito Federal & $\mathbf{4 6 . 1}$ & $\mathbf{5 3 . 1}$ \\
Durango & $\mathbf{6 3 . 2}$ & $\mathbf{3 6 . 1}$ \\
Guanajuato & $\mathbf{4 9 . 3}$ & $\mathbf{4 9 . 2}$ \\
Guerrero & $\mathbf{6 2 . 0}$ & $\mathbf{3 7 . 4}$ \\
Hidalgo & $\mathbf{4 9 . 5}$ & $\mathbf{5 0 . 3}$ \\
Jalisco & $\mathbf{4 8 . 2}$ & $\mathbf{5 0 . 8}$ \\
Mexico & $\mathbf{5 3 . 5}$ & $\mathbf{4 6 . 3}$ \\
Michoacán & $\mathbf{5 6 . 4}$ & $\mathbf{4 3 . 4}$ \\
Morelos & $\mathbf{5 1 . 5}$ & $\mathbf{4 8 . 3}$ \\
Nayarit & $\mathbf{6 7 . 3}$ & $\mathbf{3 2 . 2}$ \\
Nuevo León & $\mathbf{3 4 . 0}$ & $\mathbf{5 3 . 4}$ \\
Oaxaca & $\mathbf{5 2 . 2}$ & $\mathbf{4 7 . 5}$ \\
Puebla & $\mathbf{5 0 . 3}$ & $\mathbf{4 9 . 6}$ \\
Querétaro & $\mathbf{4 9 . 9}$ & $\mathbf{4 9 . 2}$ \\
Quintana Roo & $\mathbf{5 7 . 2}$ & $\mathbf{4 2 . 1}$ \\
San Luis Potosi & $\mathbf{6 9 . 1}$ & $\mathbf{2 9 . 4}$ \\
Sinaloa & $\mathbf{4 8 . 6}$ & $\mathbf{5 1 . 4}$ \\
Sonora & $\mathbf{5 2 . 8}$ & $\mathbf{4 6 . 8}$ \\
Tabasco & $\mathbf{5 3 . 7}$ & $\mathbf{4 6 . 1}$ \\
Tamaulipas & $\mathbf{4 7 . 8}$ & $\mathbf{5 0 . 8}$ \\
Tlaxcala & $\mathbf{4 9 . 6}$ & $\mathbf{5 0 . 3}$ \\
Veracruz & $\mathbf{5 5 . 0}$ & $\mathbf{4 4 . 6}$ \\
Yucatán & $\mathbf{4 8 . 0}$ & $\mathbf{5 1 . 9}$ \\
Zacatecas & $\mathbf{6 6 . 7}$ & $\mathbf{3 2 . 2}$
\end{tabular}

Fuente: Elaboración propia basada en información de la base de datos de nacimientos, SINAC 2013.

El sistema de salud mexicano está compuesto por la seguridad social, los servicios a la población abierta y los servicios médicos privados. La seguridad social es atendida por instituciones como el Instituto Mexicano del Seguro Social (IMSS) que atiende a los empleados de obreros y empleados de la iniciativa privada, el Instituto de Seguridad y Servicios Sociales de los Trabajadores del Estado (ISSSTE), la Secretaría de la Defensa Nacional (SEDENA) a los militares y Petróleos Mexicanos (PEMEX) a los empleados de la industria petrolera, todos ellos están cubiertos con siete seguros incluyendo el seguro médico. La población abierta es atendida en unidades de la SSA, quien a principios de 2003 puso en marcha el Seguro Popular (SP), como una política de salud que buscaba afiliar a la población sin seguridad social, brindar protección financiera y asegurar el acceso a los servicios de salud a través de un seguro médico. La atención del SP se brinda en hospitales de la SSA y los regímenes estatales.

En México, así como ha incrementado el parto por cesárea, también se ha observado un incremento en la afiliación a los servicios de salud, ya que en México en el año 2000 tan solo 40 \% de la población se encontraba afiliada, incrementando a 47\% en 2005. En 2010 la cifra llegó a 65 \%, contando con SP 23 \% de la población; para 2015, 82 \% de la población contaba con afiliación a un seguro, y de 
ese universo, 49,9 \% correspondía al SP, $39 \%$ al IMSS, 7,7 \% al ISSSTE y $6 \%$ a las demás instituciones de salud. Durante ese proceso de afiliación, para el año 2000, 37 \% de los nacimientos esperados ocurrían al margen de los servicios de salud ${ }^{7}$ y para 2014 esta cifra se redujo $25 \%$. Es decir, en catorce años se incrementó la atención institucional del parto 119 \% y en la misma proporción el número de cesáreas. ${ }^{8}$

El incremento en la medicalización del parto ha estado asociado con el interés del Estado mexicano en disminuir la MM (Freyermuth y Luna, 2014) y en incrementar la atención institucional del embarazo, sin embargo, no se tiene conocimiento de estudios que documenten que características sociodemográficas de la mujer y que factores institucionales pueden asociarse con la presencia de la cesárea durante el parto. En ese sentido, el objetivo de este artículo es describir y analizar la prevalencia del parto por cesárea en México para el periodo 2011-2014, con la finalidad de explorar cuáles fueron los factores que favorecieron la presencia de este tipo de parto.

\section{Material y Métodos}

Para llevar a cabo el análisis se empleó un estudio de tipo transversal, observacional y descriptivo, mediante el cual analizamos los eventos de cesárea durante el parto, que se encontraron en las bases de datos de nacimientos de 2011 a 2014 dentro de la República Mexicana.

Las bases de datos de nacimientos son puestas a disposición del público usuario por parte de la Dirección General de Información en Salud (DGIS) mediante un Subsistema de Información sobre Nacimientos (SINAC) $)^{9}$ que se alimenta del certificado de nacimientos y capta la información de los nacidos vivos ocurridos durante el año dentro del país.

Este subsistema incluye información sobre las condiciones del nacimiento con una cobertura nacional y su principal limitante es la mejora en la captación de los nacidos vivos fuera de las unidades médicas. Por tanto, para mejorar la calidad de la información, recurre al cálculo de factores de ajuste con la finalidad de minimizar los errores que parten del subregistro de la información.

Utilizamos también información del Índice de Desarrollo Humano (IDH) proveniente del Programa de las Naciones Unidas para el Desarrollo (PNUD, 2014), que proporciona información acerca del

7 Nacimientos ocurridos en vía pública o en el hogar

8 Cálculos a partir de información de los Boletines de la DGIS/SSA, 2015 y las proyecciones de nacimientos en México, 1990-2030 de CONAPO, Censo 2010.

9 Las bases de datos del subsistema se encuentran disponibles en línea: http://www.dgis.salud.gob.mx/contenidos/basesdedatos/std_nacimientos.html. 
nivel de desarrollo de los países. Otra información externa adicionada al análisis es el porcentaje de Hablantes de Lengua Indígena (HLI) por municipio, información proveniente del Sistema de Integración Territorial (ITER) 2010 del INEGI. Este índice es importante porque una parte de la población mayor a 3 años de algunos estados, como Chiapas (27\%) es HLI. Esta característica puede implicar una diferenciación en el acceso a los servicios de salud y, por tanto, al tipo de parto por ejemplo, de acuerdo con los usos y costumbres, las mujeres indígenas prefieren atender su parto en el hogar (Cordero, 2010. p.p. 15-16).

\subsection{Variables}

La selección de variables se realizó en primera instancia, partiendo de un análisis previo que se llevó a cabo para el estado de Chiapas de donde las variables y su codificación se retoman (Freyermuth, Muños y Ochoa, 2017). Se realizó la integración de las bases de datos de los años 2011 a 2014 en una única base de datos, donde se identificaron las variables del estado conyugal de la madre, la escolaridad, la edad, derechohabiencia, el trimestre de inicio de control prenatal, el total de consultas prenatales, si el parto fue único o múltiple, el peso del recién nacido, las semanas de gestación, el sitio del parto, el orden de nacimiento y los días de estancia Esta última como una forma de acercamiento, porque la presencia de cesárea incrementa la posibilidad de aumentar la estadía hospitalaria en diferentes niveles.

La codificación final de las variables se puede observar en la tabla 1. Para las variables externas, la clasificación corresponde a la establecida por el PNUD: bajo, medio bajo, medio alto y alto, y en el análisis se utiliza como variable continua. La variable Presencia Indígena se codifica en tres categorías: población indígena, con presencia indígena y con población indígena dispersa, según municipio. Estas categorías se formulan en el sentido de mayor a menor presencia de población indígena, en ese orden.

\subsection{Análisis estadísticos}

Como primer paso en el análisis, se calcularon estadísticas descriptivas que nos permitieron revisar las características sociodemográficas de las mujeres, del recién nacido y la atención recibida en los servicios de salud. En segundo lugar, se empleó un modelo de regresión logística de efectos mixtos y uno de efectos aleatorios para los grupos, debido a que dentro de las observaciones se presenta un problema de correlación, derivado de la estructura jerárquica respecto a la variable municipal. Lo anterior implica que, en este tipo de datos, las respuestas o características de individuos pertenecientes a un mismo grupo generalmente son más similares que las de grupos diferentes. 
Este hecho puede distorsionar los efectos del grupo y así mismo las conclusiones que se obtengan del análisis.

Este tipo de modelo permite observar puntualmente en qué medida las variables o factores analizados incrementan o disminuyen las posibilidades de que un parto sea llevado a cabo mediante cesárea ${ }^{10}$. Para el análisis de la presencia de cesárea, la codificación de la variable dicotómica de respuesta fue 1 si el parto fue por cesárea y 0 en caso contrario. El total de casos utilizados por el modelo fue de 5495 236. Para la codificación de las variables independientes se descartaron los casos correspondientes a respuestas "No especificado" o "Se ignora". Este análisis se llevó a cabo con el programa STATA 11.

\section{Resultados}

\subsection{Análisis descriptivo}

Los resultados del análisis descriptivo se presentan en la tabla 1 (al final del documento), donde se observa que, en el estado conyugal de la madre, el porcentaje de cesárea se ubica alrededor de 45 \% para cada una de las categorías, por lo que no hay diferencia entre ser alguna vez unida, unida o soltera respecto al porcentaje de cesárea.

Un factor importante es la escolaridad de la madre; en esta característica se observa que, a mayor escolaridad, mayor porcentaje de cesáreas. Resalta la enorme diferencia entre ninguna escolaridad o primaria incompleta $(31,08 \%)$ y profesional o posgrado $(70,43 \%)$, con casi cuarenta puntos porcentuales de diferencia respecto al porcentaje de cesáreas.

Al igual que la escolaridad, el porcentaje de cesáreas incrementa conforme incrementa el rango de edades. Aun cuando la diferencia entre el último rango y el primero no es tan notoria como en el factor anterior, si hay una diferencia considerable entre las madres menores de 18 años $(36,86 \%)$ y las de 35 a 44 (57,48 \%), de aproximadamente 20 puntos porcentuales.

En torno a la derechohabiencia de la madre, se observa que el IMSS-Oportunidades y el Seguro Popular, dos instituciones que atienden principalmente a la población abierta (que no cuentan con todos los seguros de la seguridad social pero que tienen un seguro médico con una cobertura explícita de intervenciones), sean las que presentan los más bajos porcentajes de cesáreas. En

\footnotetext{
10 Una mayor descripción se puede encontrar en el artículo: Freyermuth María Graciela, José Alberto Muños y Pilar Ochoa. 2017 "From therapeutic to elective cesarean deliveries: factors associated with the increase in cesarean deliveries in Chiapas". In International Journal for Equity in Health. 16:88. Pp. 2-15. DOI 10.1186/s12939-017-0582-2.
} 
cambio, la población de madres que cuentan con seguridad social tiene un porcentaje mayor al 50 $\%$, como en el caso del IMSS, PEMEX, SEDENA o SEMAR. Específicamente, el mayor porcentaje de cesáreas se presenta en las mujeres con afiliación al ISSSTE con casi $75 \%$.

En torno al trimestre de inicio de control prenatal y el total de consultas, se observa que el mayor porcentaje de cesáreas se presenta cuando el inicio de consultas prenatales es desde el primer trimestre y cuando el número de consultas rebasa las seis consultas prenatales. En los demás casos, la presencia de cesárea se ubicó por debajo del $36 \%$.

Respecto al tipo de producto, el porcentaje de cesáreas para cuando el producto es único es de 45 $\%$, elevado si consideramos que este porcentaje es de $85,8 \%$ cuando el producto es múltiple, que son partos que posiblemente ameriten un mayor cuidado durante la labor de parto con respecto al producto único.

En cuanto a las semanas de gestación, el porcentaje de cesáreas sobrepasa el 40 \% en todas las categorías, excepto en la de menos de 28. La mayor ocurrencia se observa para el grupo de 28 a 36 semanas con casi $65 \%$.

La variable sitio de parto muestra que, cuando se trata del IMSS-Oportunidades o de la Secretaría de Salud (SSA), el porcentaje de cesáreas es bajo en comparación con instituciones como el IMSS (46,97 \%) O PEMEX, SEDENA o SEMAR (49,16\%), también respecto a otra unidad pública (47,93 \%), todos por encima del $45 \%$. Los casos sobresalientes son el del ISSSTE con un porcentaje cercano al $70 \%$ y las unidades médicas privadas donde el porcentaje llega a casi $80 \%$. Es decir, en el ISSSTE siete de cada diez mujeres que atienden su parto en la institución lo realizan vía cesárea. Es más alarmante aun en las privadas donde ocho de cada diez partos terminan en cesárea.

Si observamos el orden de nacimiento como una variable que incluye del primer nacimiento hasta 11 o más nacimientos, el porcentaje de cesáreas disminuye conforme se incrementa dicho orden. Es decir, el porcentaje de cesáreas es mayor $(48,46 n \%)$ cuando se trata del primer o segundo nacimiento que si se trata del tercer a quinto nacimiento; porcentaje que a su vez es mayor que el obtenido en el sexto a décimo nacimiento y que el grupo que va del onceavo nacimiento o más.

En términos generales, el porcentaje de los días de estancia en torno a la presencia o no de cesárea durante el parto se incrementa en los rangos inmediatos a la categoría de 0 a 1 días, es decir, el incremento porcentual se observa entre 2 a 7 días, 8 a 30 y 31 a 99 días en 4,5 puntos porcentuales. Dicho porcentaje se mantiene entre $45 \%$ y 50 \% y el porcentaje más alto se sitúa entre el mes y los tres meses de hospitalización (49,55\%).

No hay una diferencia notable entre las poblaciones con presencia indígena y con población indígena dispersa respecto a la presencia de cesárea durante el parto. Sin embargo, donde 
observamos una mayor diferencia es entre las categorías con presencia indígena y población indígena, que alcanza los 15 puntos porcentuales, siendo mayor la segunda.

Finalmente, en la distribución de la variable IDH, se observa un mayor porcentaje de cesáreas con una diferencia bastante amplia entre las categorías bajo $(20,18)$ y alto $(53,42)$, de 33 puntos porcentuales.

\subsection{Modelo de regresión logística}

Los resultados del modelo logístico se observan en la Tabla 2 (Al final del documento). El primer resultado del modelo, correspondiente a la variable estado conyugal de la madre, no muestra significancia estadística para las categorías unida y soltera con respecto a las mujeres alguna vez unidas.

En cambio, para la escolaridad se observa la misma dinámica que en los porcentajes de cesárea; es decir, con respecto a ninguna escolaridad o primaria incompleta como categoría de comparación, el riesgo de tener una cesárea durante el parto se incrementa conforme aumenta la escolaridad. Esto significa que comparado con las mujeres que no concluyeron la primaria o no fueron a la escuela, las posibilidades de atenderse por cesárea durante el parto para las de mayor escolaridad -profesional o posgrado-son $50 \%$ mayores.

En el caso de la edad, la tendencia es la misma que el resultado anterior, conforme aumenta la edad se incrementan las posibilidades de atender el parto por cesárea. Las mujeres que tenían entre 18 y 24 años tenían un $3 \%$ más de tener una cesárea que el grupo de referencia (menores de 18 años); en cuanto a las mujeres de 25 a 34 años, sus posibilidades se incrementan $3 \%$ más que el grupo de referencia, y finalmente, las posibilidades son mayores si observamos el grupo de 35 a 44 con 2 veces más posibilidades, casi lo mismo que para el grupo de 45 o más.

En lo referente a la derechohabiencia, si consideramos ninguna como categoría de referencia, todos los resultados son significativos, excepto para el IMSS-Oportunidades. En todos los casos se tiene una mayor probabilidad de cesárea (alrededor de 20 \%) salvo para el Seguro Popular, cuyo resultado disminuye las posibilidades de cesárea en un $2 \%$.

En cuanto al trimestre de inicio del control prenatal, el modelo sugiere que hay una mayor posibilidad de cesárea para las madres que iniciaron el control durante el primer trimestre respecto a aquellas que nunca asistieron a control prenatal. En cambio, para las que lo iniciaron durante el segundo o tercer trimestre, los resultados no son significativos. Con relación al total de consultas prenatales, la información que arroja el modelo indica que, a mayor número de consultas, aumenta la posibilidad de una cesárea, respecto a haber asistido a 1 o ninguna. La propensión aumenta un $20 \%$ para las que tuvieron entre 2 y 5 consultas con respecto al grupo de referencia y las 
posibilidades de cesárea se incrementan aún más para las madres que acudieron a 6 o más consultas.

El parto múltiple es sin duda un determinante asociado a la presencia de cesáreas dentro de nuestro análisis. El modelo nos da información referente a la posibilidad de tener una cesárea si el parto es múltiple o no. Los resultados indican que la propensión por cesárea, cuando es un parto múltiple, se incrementa en 6,18 veces más que cuando no lo es. El peso del recién nacido también parece ser determinante para la elección de la cesárea cuando este es menor a $2500 \mathrm{gr}$ o de $4000 \mathrm{gr}$ y más, con respecto al peso entre 2500 y 3999 gr (grupo de referencia).

Respecto a las semanas de gestación, el tener menos de 28 semanas se observa como un factor protector con respecto al grupo de referencia (de 37 a 41 semanas). Es decir, tener ese número de semanas a la hora del parto reduce la posibilidad de que sea terminado vía cesárea. En cambio, para los partos dentro de las 28 a 36 semanas, se tiene dos veces mayores posibilidades de cesárea. Para los partos dentro de las 42 a 45 semanas, se tiene 0,6 veces más posibilidades que el grupo de referencia.

La atención del parto y la presencia de cesáreas dependen bastante del lugar de atención. En este caso, cuando la atención es en el IMSS-Oportunidades, se reducen las posibilidades de tener una cesárea $30 \%$, respecto de la SSA. En cambio, si comparamos la atención entre SSA y el IMSS, se tiene que la presencia de cesáreas en este último aumenta $8 \%$; para el ISSSTE esta misma posibilidad es 1,20 veces mayor; en el caso de PEMEX, SEDENA o SEMAR la posibilidad de tener una cesárea es alrededor de $13 \%$ mayor, y para otra unidad pública aumenta alrededor de $60 \%$. El mayor incremento en las posibilidades de cesárea dentro de estas categorías, respecto de la atención en la SSA, se da en las unidades médicas privadas, donde se observa 5 veces más posibilidades de que el parto sea por esa vía.

En cuanto al orden de nacimiento, el resultado para los nacimientos subsecuentes al primer nacimiento resultó ser un factor que disminuye la propensión de tener una cesárea durante el parto, además, la propensión se incrementa considerablemente conforme aumenta el número de nacimientos.

Por otro lado, ante la presencia de una cesárea, los días de estancia resultan relevantes si suponemos que es posible que ante una cesárea se incrementen los días de estancia hospitalaria, pues ello en términos de costos a la institución le afectaría. Los resultados del modelo muestran que si consideramos la presencia de cesárea respecto a la primera categoría de días de estancia ( 0 a 1$)$ es un factor que incrementa 1,09 veces más la posibilidad de una mayor estadía ( 2 a 7 días) y 1,08 veces más probable en torno a los 8 a 30 días, salvo para la categoría 31 a 99 días que no resulta significativa en el modelo. 
En torno a los lugares con presencia indígena, la propensión de cesáreas se incrementa en 12,5\% si se trata de un lugar con población indígena. El hecho de que se traté de una localidad o municipio con presencia indígena dispersa también incrementa la posibilidad de cesárea respecto a que haya presencia indígena, aunque en un porcentaje menor, cercano al $6 \%$.

Finalmente, los resultados del modelo indican que la posibilidad de tener una cesárea es más alta en los municipios con un mayor IDH. Es decir, considerando aquellos municipios con un IDH bajo como categoría de referencia, el riesgo de una cesárea se observa alrededor de 34 \% mayor para aquellos donde el IDH está en el rango medio bajo, se incrementa para los que están en medio alto $(0,68$ veces $)$ y para los de IDH alto $(0,78$ veces más posibilidades $)$.

\section{Discusión}

En el presente trabajo se analiza qué factores tienen una influencia importante sobre la presencia de cesárea durante el parto en México. Los resultados del análisis confirman los hallazgos de un análisis previo que se realizó para el estado de Chiapas (Freyermuth, Muños y Ochoa, 2017) y añade diferencias a nivel nacional respecto a algunas de las categorías en los factores como la derechohabiencia, las consultas de control prenatal, la población indígena, el IDH y el sitio de parto.

Específicamente, entre las diferencias se observa que, en los resultados obtenidos para el caso de Chiapas, atender el parto bajo el SP era un factor de riesgo de tener una cesárea, respecto a la ausencia de afiliación. En cambio, para el presente análisis el resultado no se considera de tal manera Un elemento diferencial es que en Chiapas la atención médica se ha realizado fundamentalmente a través del SP (80 \% en HLI y $68,3 \%$ en toda la población). A nivel nacional la cobertura es para HLI de $73 \%$ y para toda la población es de $41 \%$. El caso de Chiapas también es suigéneris en que el porcentaje de $\mathrm{HLI}$ es de $28 \%$ y a nivel nacional constituyen el 6,5 \% (Luna y Freyermuth, 2019). Adicionalmente, en la última década, Chiapas estuvo en los primeros puestos de mortalidad materna lo que llevó a que en este junto con otros ocho estados se focalizaran las políticas de salud dirigidas a disminuir este problema (Freyermuth y Luna, 2014). Esto aunado a que, en el caso de los servicios públicos, se cuenta con un sistema de aseguramiento entre la población abierta, conocido como el $\mathrm{SP}$, que al igual que los seguros médicos, paga diferencialmente el doble por el procedimiento de cesárea. Como antecedente en México, se sabe que, desde el ingreso de los seguros médicos privados, la cesárea se incrementó exponencialmente, lo que hace evidente que hay una relación costo-beneficio para el mercado de la salud. 
Además, algunos servicios del SP pueden ser subrogados al sector privado (Leal-Fernández, 2013). En México, generalmente, las familias de más bajos ingresos son las que están siendo afiliadas a este seguro (Meneses-Navarro, 2007), por lo que las mujeres de más bajos recursos quedan exentas del pago por los servicios obstétricos, sea cual sea el procedimiento. La diferencia entre los dos escenarios (Chiapas y México) puede radicar en que, en el primero casi el $100 \%$ de la población objetivo se encontraba afiliada al SP en 2014, y en el total de la república, para el mismo año, solo el $50 \%$ de la población.

Este panorama podría asociarse con el hecho de que haber recibido control prenatal desde el primer trimestre, incrementa el riesgo de cesárea versus no haberlo recibido. Puesto que el no haber llevado su control prenatal en alguna de las instituciones de salud no afecta el hecho de que el parto sea atendido vía institucional, derivado de la puesta en marcha del programa de salud materna y perinatal en México, que permite la atención del parto aun cuando la madre no sea beneficiaría de la institución. Así, la mayoría de estas mujeres puede decidir si acudir a una institución de salud o atenderse en casa con métodos tradicionales. En ese sentido, se puede decir que la medicalización del parto tiene como uno de sus resultados el incremento en las cesáreas, lo que se reafirma con el hallazgo de una asociación positiva entre el número de consultas prenatales y la tasa de cesáreas.

La resolución de los partos por cesáreas también está relacionada con otros factores como el nivel de escolaridad y la calidad de vida, ya que cuando estos son altos, son posibles causas para que las mujeres sean más proclives a esta forma de resolución del parto. Otro posible factor, aunque controversial, es que en la medida en que se tiene la percepción de que es la forma más segura, fácil y rápida para tener un hijo, prefieran terminarlo por cesárea, considerando al parto vaginal como un método anticuado (González, Vega, Cabrera, Muñoz y Valle, 2001; Penna y Arulkumaran, 2003).

De esta forma, se ha observado que los principales argumentos para la decisión de realizarse una cesárea son programar la fecha exacta para facilitar la presencia de la familia y del padre del recién nacido, así como la planeación del cuidado de los hijos más grandes y evitar un trabajo de parto doloroso que puede durar de 6 a 10 horas. Todo lo anterior gracias a las nuevas tecnologías que, además de permitir el conocer con bastante certeza el sexo del producto y por lo tanto asignarle un nombre, hacen posible establecer el grado de madurez fetal facilitando la asignación de una fecha de nacimiento, justificaciones que pueden verse fortalecidas por las prácticas de los profesionales de la salud (Plante, 2006; Farland, 2009; Villanueva, 2004; Queenan, 2004).

El hecho que el primer o segundo parto ocurra mediante cesárea podría implicar una nueva estrategia para resolver la sobrecarga hospitalaria en los establecimientos públicos de ginecoobstetricia y con la intención de evitar que se rechace la hospitalización cuando acuden sin un trabajo de parto efectivo. Por un lado, se cuenta con un programa específico de salud materna 
y perinatal, que define estrategias entre las cuales se tiene el Convenio General de Colaboración Interinstitucional de Emergencias Obstétricas. Esta permite obtener atención en cualquiera de las instituciones de prestación de servicios de salud sin ser beneficiaria del servicio (Gobierno Federal, 2013). Por otro lado, se presentan altos niveles de saturación entre los hospitales gubernamentales, pertenecientes a la Secretaría de Salud y a los estados, que son los principales centros de atención del SP (Observatorio del Desempeño Hospitalario, 2011).

Un antecedente de importancia, es que desde 1998-2018 las mujeres que recibían transferencias monetarias a través de un programa de bienestar social tenían como corresponsabilidad acudir a la atención prenatal y atenderse su parto intrahospitalariamente. En México 5 de cada 10 familias contaban con algún programa social y desde el 2004 se instaura el SP, pero podemos señalar que en las regiones rurales este programa se consolidó hasta la segunda década del siglo XXI, por ello que el incremento de cesáreas esté ocurriendo en las generaciones más jóvenes. Por otro lado, desde el 2001 al 2015 los servicios de salud públicos han tendido un aumento tanto de ginecoobstetras (50\%), de camas censables de ginecología (30\%), incubadoras (30\%) y bancos de sangre (23\%) (SINERHIAS, 2018); sin embargo, las salas de expulsión han disminuido tres veces en 2015 con respeto a las que se tenían en 2001 y la población no derechohabiente disminuyó de 57,81 a $15,1 \%$ (Luna y Freyermuth 2019). Es decir, que se ha incrementado la demanda de servicios, pero las salas de expulsión han disminuido.

A pesar de lo anterior, el costo de la cesárea resulta más elevado no solamente para las instituciones por los insumos y equipo médico que se utilizan, sino también para la población debido a todas las complicaciones a corto, mediano y largo plazo que pueden aparecer en las mujeres sometidas a este procedimiento. Pues es 1,09 veces más probable que las mujeres permanezcan de 2 a 7 días en el hospital por cesárea y 1,08 veces más probable que su estancia sea entre 8 y 30 días por la misma causa que permanecer entre 0 y 1 días.

Al quedarse hospitalizadas, se adquieren riesgos en la hospitalización, entre los que destacan la infección de la herida quirúrgica derivándose de esta múltiples complicaciones (Henderson y Love, 1995; Rosales y Felguérez, 2009). También, se ha asociado a ruptura uterina derivando en fístula vesicouterina (Joźwik y Lotocki, 1997) y a deformaciones que causan infertilidad (Cárdenas, 2002). La cesárea es considerada también un factor de riesgo de hemorragia porque propicia la presentación de placenta previa, acretismo placentario, desprendimiento prematuro de placenta y ruptura uterina (Secretaría de Salud, 2009b) en embarazos ulteriores. La morbilidad poscesárea también afecta a los recién nacidos, puesto que se relaciona con varios tipos de complicaciones respiratorias (Cárdenas, 2002), además se ha encontrado una alteración del grado de metilación del ADN de genes específicos en los descendientes, asociado esto al número de horas de labor (Malin et al, 2014). 
En cuanto a los municipios y la presencia indígena, la atención del parto conduce a una mayor posibilidad de cesárea si los municipios cuentan con una mayoría de población indígena. Si consideramos que en nuestro país la población indígena presenta los índices más altos de pobreza y marginación (Consejo Nacional de Evaluación de la Política Social, 2015), este explicaría una de las razones del porqué las mujeres indígenas son renuentes a asistir a los hospitales. Un estudio a nivel nacional revela que la RMM es de 85 en municipios con $70 \%$ o más de $\mathrm{HLI}$, a diferencia de municipios con menos de $40 \%$ y una RMM de 37 (Freyermuth, 2016). Es decir, de casi el doble. Aunque hay que señalar que del 2008 al 2017, la mortalidad en mujeres indígenas ha disminuido, de una RMM de 171 a 78, de manera más acelerada (55 \%) que en el resto de las mujeres (34 \%) de una RMM de 49,8 a 33,1 (Freyermuth,2019). Ante estos resultados, es posible que las dificultades de acceso de las mujeres indígenas a los servicios hospitalarios determinen que se estén realizando cesáreas bajo el término de "por razones necesarias", a fin de evitar que las pacientes sin un trabajo de parto activo sean rechazadas de los servicios y no retornen.

Las mujeres que viven en situación de pobreza tienen menos posibilidades de decidir la forma en que será resuelto el nacimiento y es poco frecuente que se les informe sobre los riesgos de la cesárea. Por ello, se ha considerado que cuando esta práctica es innecesaria e injustificada puede verse como una transgresión a los derechos reproductivos de las mujeres, debido a que es admitida parcial o completamente por el Estado al incumplir su deber de "respetar, proteger y garantizar los derechos humanos que los conforman" (Facio, 2008, p. 13). Chiapas y el Distrito Federal son las únicas entidades que contemplan a los derechos reproductivos en su Ley de Acceso de las Mujeres a una Vida Libre de Violencia. En el caso de Chiapas la violación de este derecho se define como:

Toda acción u omisión que limite o vulnere el derecho de las mujeres a decidir libre y voluntariamente sobre su función reproductiva, en relación con el número y espaciamiento de los hijos, acceso a métodos anticonceptivos de su elección, acceso a una maternidad elegida y segura, así como los servicios de atención prenatal, y obstétricos de emergencia (Ley de Acceso a una Vida Libre de Violencia para las Mujeres en el Estado de Chiapas, 2009, p. 6).

Controversialmente, la práctica de la cesárea injustificada cae dentro de lo que se ha llamado violencia obstétrica, que ya ha sido definida y considerada en las leyes de acceso de nueve estados de la República Mexicana. En cuanto a la penalización de este tipo de violencia, Chiapas y Veracruz son las únicas entidades que hasta el momento tienen tipificada en sus códigos penales la violencia obstétrica como tal. En otras entidades como Baja California Sur, ${ }^{11}$ Campeche, ${ }^{12}$ Chihuahua, $^{13}$

Artículo 343, fracc. I del Código Penal de Baja California Sur.

Artículo 276, fracc. I del Código Penal de Campeche.

Artículo 320, fracc. I del Código Penal de Chihuahua. 
Distrito Federal, ${ }^{14}$ Durango ${ }^{15}$ y Tabasco $^{16}$ existe la tipificación de un delito que hace alusión a la responsabilidad técnica de la persona médica, en el cual se sancionan las operaciones quirúrgicas innecesarias. En este delito podría caber la práctica de la cesárea cuando hay posibilidad de realizar un parto vaginal sin riesgo; dicho delito se sanciona con alrededor de 2 a 7 años de prisión en estos estados.

Dentro de las agrupaciones médicas, en la FEMECOG existe polémica y desacuerdo respecto a la definición de violencia obstétrica y a su sanción penal. Se argumenta que sus procedimientos son llevados a cabo responsablemente con una validación científica, con la intención de obtener el mejor resultado y beneficio de la madre y el recién nacido. Sin embargo, señala en un pronunciamiento emitido en junio de 2015 que:

Las condiciones de la actual infraestructura hospitalaria en nuestro país no permiten realizar prácticas de acompañamiento durante el trabajo de parto y nacimiento, que serían deseables. Dicha infraestructura es uno de los principales factores que intervienen en este complejo proceso y los ginecoobstetras somos totalmente ajenos a ella. (Secretaría de Salud, 1995, p. 2)

En su pronunciamiento, la FEMECOG acepta que se está realizando este procedimiento de manera electiva por las condiciones laborales $y$, por tanto, la violación a los derechos de las mujeres correspondería directamente al Estado mexicano. Sin embargo, este argumento, como señala un pronunciamiento del Observatorio de Mortalidad Materna (OMM, 2015) es débil, ya que el mayor porcentaje de partos por cesárea con respecto al parto vaginal se realiza en la práctica médica privada (88 \% en Chiapas y $80 \%$ a nivel nacional en 2014), ${ }^{17}$ en la cual estos especialistas sí tendrían la posibilidad de realizar el acompañamiento durante el trabajo de parto y nacimiento. La FEMECOG propone que en lugar de emitir sanciones habría que apelar por mejor infraestructura, capacitación y recursos humanos para llevar a cabo la atención integral, tan importante, correspondiente al posparto.

\section{Conclusiones}

Artículo 326, fracc. I del Código Penal del Distrito Federal.

Artículo 236, fracc. I del Código Penal de Durango.

Artículo 341, fracc. I del Código Penal de Tabasco.

Información del SINAC/SINAIS/DGIS/SSA, Cubos, México, 2015. 
Es importante estudiar a profundidad los mecanismos mediante los cuales el nuevo sistema de aseguramiento (Seguro Popular) está incrementando el número de cesáreas en los hospitales públicos del país, ya que podría ser un factor estructural que suma a una gran cantidad de mujeres a someterse a cesáreas innecesarias, pese a que no cuenten con las particularidades de aquellas que históricamente han tenido riesgo más elevado y necesitan este procedimiento.

Este estudio resalta la necesidad de llevar a cabo acciones en distintos ámbitos, ya que la cesárea injustificada es un problema multicausal. Se hace evidente la ausencia de organización institucional y se observa que es necesaria la regulación del personal médico, para que se efectúe una cesárea de acuerdo con protocolos institucionales, leyes estatales y federales. Dicho fenómeno se piensa multicausal, pues, además de ciertas características sociodemográficas de las mujeres, las razones clínicas y las decisiones médicas, los nuevos sistemas de afiliación a los servicios de salud públicos podrían estar contribuyendo al incremento de las cesáreas.

El incremento en la demanda de los servicios de salud materna, inducida a través de los programas de trasferencias monetarias con corresponsabilidades y la puesta en marcha del SP, no han ido seguidas de una política de aumento de las salas de expulsión, lo que favorece el incremento de cesáreas.

Específicamente, se confirma una relación directa entre número de consultas prenatales y cesáreas; así como el trimestre de inicio de atención y la mayor posibilidad de cesárea. También se reconoce que hay mayor posibilidad de resolución de un parto por cesárea en mujeres con mayor escolaridad y que viven en municipios con mayor índice de desarrollo humano, así como el habitar en municipios predominantemente indígenas. Nuestro modelo también determina que la presencia de una cesárea incrementa la probabilidad de un aumento en los días de estancia. Por otro lado, se registra que ante la presencia de cesárea para la cohorte del primer y segundo nacimiento se reduce la propensión en los subsecuentes.

\section{Referencias}

Araujo, F. J., Encinas, C., Torres, M. A. y Caballero, M. V. (2011). Asepsia y antisepsia. Visión histórica desde un cuadro. Apuntes de Ciencia, (2), 60-64. Recuperado de http://apuntes.hgucr.es/2011/06/27/asepsia-y-antisepsia-vision-historica-desde-un-cuadro/

Becerro de Bengoa-Callau, C. y Becerro de Bengoa-García, C. (2001). Analgesia y anestesia en obstetricia. Toko-Ginecología Práctica, 60(2), 80-94. 
Belloso, H. (2009). Historia de los antibióticos. Revista del Hospital Italiano de Buenos Aires, 29(2), 102-11.

Betrán, A. P., Torloni, M. R., Zhang, J., Ye, J. Mikolajczyk, R., Deneux-Tharaux, C., Oladapo, ... Gülmezoglu, A. M. (2015). What is the optimal rate of caesarean section at population level? A systematic review of ecologic studies. Reproductive Health, 12(10), 57. DOI: 10.1186/s12978-015-0043-6

Betrán, A. P., Merialdi, M., Lauer, J. A., Bing-Shun, W., Thomas, J., Look, P. V. y Wagner, M. (2007). Rates of caesarean section: analysis of global, regional and national estimates. Paediatric and Perinatal Epidemiology, 21(2), 98-113. DOI: 10.1111/j.1365-3016.2007.00786.x

Cárdenas, R. (2002). Complicaciones asociadas a la cesárea: la importancia de un uso módicamente justificado. Gaceta Médica de México, 138(4), 357-66. Recuperado de https://www.medigraphic.com/cgi-bin/new/resumen.cgi?IDARTICULO=7470

Celesia, C. (2004). Breve historia de la analgesia en obstetricia. Revista del Hospital Materno Infantil Ramón Sardá, 23(3), 122-25. Recuperado de http://www.redalyc.org/articulo.oa?id =91223307\&idp $=1$ \&cid $=1557578$

Consejo Nacional de Evaluación de la Política Social (2015). Resultados de pobreza en México 2014 a nivel nacional $y$ por entidades federativas. Recuperado de http://www.coneval.gob.mx/Medicion/MP/Paginas/Pobreza_2014.aspx

Cordero, L. (2010). Salud de la mujer indígena: intervenciones para reducir la muerte materna. Recuperado de https://publications.iadb.org/es/publicacion/14192/salud-de-la-mujer-indigena-intervencionespara-reducir-la-muerte-materna

Dirección General de Información en Salud. (2014). Base de datos de Certificado de Nacimiento-Nacimientos ocurridos 2013. Recuperado de http://www.dgis.salud.gob.mx/contenidos/sinais/s index.html. 
Dirección General de Información en Salud. (2015). Boletín de información estadística 2000-2011 (Vol. 2). México:

DGIS/SSA.

Recuperado

de http://www.dgis.salud.gob.mx/contenidos/publicaciones/p bie.html

Emmett, C. L., Shaw, A. R., Montgomery, A. A., Murphy, D. J. y Diamond Study Group (2006). Women's experience of decision making about mode of delivery after a previous caesarean section: the role of health professionals and information about health risks. BJOG, 113(12), 1438-45. DOI: 10.1111/j.1471-0528.2006.01112.x

Encuesta Nacional de Salud y Nutrición (2012). Documentos analíticos. Elevada recurrencia a las cesáreas: revertir la tendencia y mejorar la calidad en el parto. Recuperado de http://ensanut.insp.mx/documentos analiticos.php\#.VY2LlvmG EY

Facio, A. (2008). Necesidad de enmarcar la salud sexual y reproductiva en un marco de derechos humanos. En Instituto Interamericano de Derechos Humanos (Ed.), Los derechos reproductivos son derechos humanos (pp. 13-17). San José, Costa Rica: IIDH.

Farland, L. V. (2009). The use and overuse of cesarean sections in Mexico. TuftScope, 9(1), 30-33.

Federación Mexicana de Colegios de Obstetricia y Ginecología. (2015). Pronunciamiento FEMECOG. Recuperado de http://www.femecog.org.mx/docs/FEMECOG\%20pronunciamiento.pdf

Freyermuth, M. G. (2016). Determinantes sociales en la mortalidad materna en México. Revista CONAMED, 21(1), 25-32. Recuperado de http://www.dgdi-conamed.salud.gob.mx/ojsconamed/index.php/revconamed/article/view/400/709

Freyermuth, M. G. (1 y 2 de abril de 2019). La partería en México desde el punto de vista de las usuarias indígenas. En Foro Diálogos Académicos y Problemas Nacionales, CIESAS, México. 
Luna, M. y Freyermuth, M. G. (2019). Población hablante de lengua indígena en México: indicadores sociodemográficos 2015. 2da edición ampliada a 17 entidades federativas [PDF]. Ciudad de México: Centro de Investigaciones y Estudios Superiores en Antropología Social.

Freyermuth, M. G., Muños, J. A. y Ochoa M. del P. (2017). From therapeutic to elective cesarean deliveries: factors associated with the increase in cesarean deliveries in Chiapas. International Journal for Equity in Health, 16(88). doi: 10.1186/s12939-017-0582-2

Freyermuth, M. G. y Luna, M. (2014). Muerte materna y muertes evitables en exceso. Propuesta metodológica para evaluar la política pública en salud. Realidad, Datos y Espacio Revista Internacional de Estadística y Geografía, 5(3), 42-61. Recuperado de https://www.inegi.org.mx/rde/2014/09/07/muerte-materna-y-muertes-evitables-en-excesopropuesta-metodologica-para-evaluar-la-politica-publica-en-salud/

Gapminder (S.F.). Razón de mortalidad materna en el periodo 1980 a 2007. Recuperado de http://www.gapminder.org/

Gómez-Dantés, O. (2004). El secuestro de Lucina (o cómo detener la epidemia de cesáreas). Salud Pública de México, 46(1), 71-74. Recuperado de http://www.scielo.org.mx/scielo.php?script=sci arttext\&pid=S003636342004000100010\&lng=es\&tlng=es

González, G. J., Vega, M. G. Cabrera, C., Muñoz, A. y Valle, A. (2001). Caesarean sections in Mexico: are there too many? Health Policy and Planning, 16(1), 62-75. DOI: 10.1093/heapol/16.1.62

Graham, W. J., Hundley, V., McCheyne, A. L., Hall, M. H., Gurney, E. y Milne, J. (marzo, 1999). An investigation of women's involvement in the decision to deliver by caesarean section. British Journal of Obstetrics and Gynaecology, 106(3), 213-20. DOI: 10.1111/j.1471-0528.1999.tb08233.x

Henderson, E. y Love, E. J. (1995). Incidence of hospital-acquired infections associated with caesarean section. Journal of Hospital Infection, 29, 245-55. DOI: 10.1016/0195-6701(95)90271-6 
Institute of Biomedical Science. (2005). A brief history of blood transfusion. Recuperado de https://www.ibms.org/go/nm:history-blood-transfusion

Instituto Nacional de Estadística y Geografía. (2013). Censo de población y vivienda 2010. Principales resultados por localidad (ITER). Recuperado de http://www.inegi.org.mx/sistemas/consulta resultados/iter2010.aspx

Joźwik, M., y Lotocki, W. (1997). Vesicouterine fistula-an analysis of 24 cases from Poland. International Journal of Gynecology and Obstetrics, 57, 169-72. DOI: 10.1016/s0020-7292(97)02837-3

Lane, T, A. y Lamkin, G. E. (1984). Paralysis of phagocyte migration due to an artificial blood substitute. Blood, 64(2), 400-05. Recuperado de http://www.bloodjournal.org/content/bloodjournal/64/2/400.full.pdf

Leal-Fernández, G. (2013). ¿Protección social en salud? Ni 'seguro', ni 'popular'. Estudios Políticos, 28, 16393. Recuperado de http://www.scielo.org.mx/scielo.php?script=sci arttext\&pid=S0185$\underline{16162013000100009}$

Ley de Acceso a una Vida Libre de Violencia para las Mujeres en el Estado de Chiapas. (23 de febrero de 2009). Diario Oficial de la Federación. México.

Malin, A., Schlinzig, T., Gómez-Cabrero, D., Gunnar, A., Sundin, M., Johansson, S., Norman, M. y Ekström, T. J. (2014). Cesarean delivery and hematopoietic stem cell epigenetics in the newborn infant: implications for future health? American Journal of Obstetrics and Gynecology, 21 1(5), 502-05.

Meneses, S. (2007). ¿Acceso universal a la atención obstétrica? El Seguro Popular de Salud frente al reto de la muerte materna en Los Altos de Chiapas. En Díaz (Ed.), Muerte materna y Seguro Popular (pp. 1364). México: Mono Comunicación S.A 
Observatorio de Mortalidad Materna. (2015). Posicionamiento del Observatorio de Mortalidad Materna frente al Pronunciamiento de la Federación Mexicana de Colegios de Obstetricia y Ginecología, A.C. en relación con la violencia obstétrica. Recuperado de http://www.omm.org.mx/omm/images/stories/Documentos\%20grandes/Posicion\%20del\%200MM -final.pdf

Organización Mundial de la Salud y Human Reproductive Program (2015). Declaración de la OMS sobre tasas de cesárea. Resumen Recuperado de http://apps.who.int/iris/bitstream/10665/161444/1/WHO RHR 15.02 spa.pdf

Penna, L. y Arulkumaran, S. (septiembre, 2003). Cesarean section for non-medical reasons. International Journal of Gynaecology and Obstetrics, 82(3), 399-409.

Pinós, T. (1997). Hazañas médicas. Barcelona, España: Planeta.

Plante, L. A. (2006). Public health implications of cesarean on demand. Obstetrical \& Gynecological Survey, 61(12), 807-15. DOI: 10.1097/01.ogx.0000248826.44123.73

Programa de las Naciones Unidas para el Desarrollo. (2014). Índice de desarrollo humano municipal en México. Recuperado de http://www.mx.undp.org/content/mexico/es/home/library/poverty/idhmunicipal-en-mexico--nueva-metodologia.html

Queenan, J. T. (2004). Elective cesarean delivery. Obstetrics and Gynecology, 103(6), 1135-36. DOI: 10.1097/01.AOG.0000128268.04368.1c

Rosales, E. y Felguérez, J. A. (2009). Repercusión demográfica de la operación cesárea. Ginecología y Obstetricia de México, 77(8), 362-66. Recuperado de https://www.medigraphic.com/pdfs/ginobsmex/gom-2009/gom098c.pdf

Sarkar, S. (2008). Artificial blood. Indian Journal of Critical Care Medicine, 12(3), 140-44. doi: 10.4103/09725229.43685 
Secretaría de Planeación, Gestión Pública y Programa de Gobierno (2014). Segundo informe de gobierno. Secretaría de Planeación, Gestión Pública y Programa de Gobierno. Recuperado de http://www.chiapas.gob.mx/informe/

Secretaría de Salud. (1995). Norma oficial mexicana sobre la atención a la mujer durante el embarazo, parto y puerperio y del recién nacido. Criterios y procedimientos para la prestación del servicio. Diario Oficial de la Federación, NOM 007-SSA2-1993.

Secretaría de Salud. (2009a). Diagnóstico y tratamiento de la hemorragia obstétrica en la segunda mitad del embarazo y puerperio inmediato. México.

Secretaría de Salud (2009b). Cesárea Segura. Subsecretaría de Prevención y Promoción de la Salud. Dirección General de Salud Reproductiva. México.

Sesia, P., García, M., García, N., Carmona, G. y Sachse, M. (2013). Resultados del monitoreo de las redes de servicios de salud materna del Estado de Oaxaca. En Freyermuth y Sesia (Ed.), Monitoreos, diagnósticos y evaluaciones en salud materna y reproductiva. Nuevas experiencias de contraloría social (pp. 181-206). México: CPMS, CIESAS, OMM.

Villanueva, L. A. (2004). Operación cesárea: una perspectiva integral. Revista de la Facultad de Medicina UNAM, 47, 246-50. Recuperado de https://www.medigraphic.com/pdfs/facmed/un2004/un046e.pdf

Villar, J., Valladares, E., Wojdyla, D., Zavaleta, N., Carroli, G., Velazco, A., ...Acosta, A. (2006). Caesarean delivery rates and pregnancy outcomes: the $2005 \mathrm{WHO}$ global survey on maternal and perinatal health in Latin America. Lancet, 367, 1819-29. DOI: 10.1016/S0140-6736(06)68704-7

Young, J. H. (1964). James Blundell (1790-1818). Experimental Physiologist and obstetrician. Medical History, $8(2), 159-69$. 


\section{Anexo. Tablas}

Tabla 1.

Factores asociados con la práctica de la cesárea, México, 2011-2014

\begin{tabular}{|c|c|c|c|c|}
\hline \multirow{2}{*}{ Características } & & \multirow{2}{*}{$\mathbf{n}$} & \multicolumn{2}{|c|}{$\%$ tipo de parto } \\
\hline & & & Vaginal & Cesárea \\
\hline \multirow{3}{*}{$\begin{array}{l}\text { Estado conyugal de la madre } \\
\qquad(\mathrm{n}=8586684)\end{array}$} & Alguna vez unida & 54319 & 55,81 & 44,19 \\
\hline & Unida & 7678479 & 53,89 & 46,11 \\
\hline & Soltera & 853886 & 55,7 & 44,3 \\
\hline \multirow{5}{*}{$\begin{array}{l}\text { Escolaridad de la madre } \\
\qquad(n=8601 \text { 101) }\end{array}$} & $\begin{array}{l}\text { Ninguna o primaria } \\
\text { incompleta }\end{array}$ & 680198 & 68,92 & 31,08 \\
\hline & Primaria completa & 1691036 & 63,23 & 36,77 \\
\hline & Secundaria completa & 3385287 & 57,79 & 42,21 \\
\hline & $\begin{array}{l}\text { Bachillerato o } \\
\text { preparatoria completa }\end{array}$ & 1684270 & 48,35 & 51,65 \\
\hline & Profesional o posgrado & 1160310 & 29,57 & 70,43 \\
\hline \multirow{5}{*}{$\begin{array}{l}\text { Edad de la madre } \\
(\mathrm{n}=8689995)\end{array}$} & Menos de 17 & 612447 & 63,14 & 36,86 \\
\hline & 18 a 24 & 3561511 & 59,62 & 40,38 \\
\hline & 25 a 34 & 3602793 & 49,9 & 50,1 \\
\hline & 35 a 44 & 897998 & 42,52 & 57,48 \\
\hline & 45 o más & 15246 & 46,72 & 53,28 \\
\hline \multirow{7}{*}{$\begin{array}{c}\text { Derechohabiencia de la } \\
\text { madre } \\
\text { (n= } 8450 \text { 170) }\end{array}$} & Ninguna & 1682772 & 44,69 & 55,31 \\
\hline & IMSS & 2153392 & 46,98 & 53,02 \\
\hline & ISSSTE & 224207 & 26,43 & 73,57 \\
\hline & $\begin{array}{l}\text { PEMEX, SEDENA O } \\
\text { SEMAR }\end{array}$ & 80821 & 47,73 & 52,27 \\
\hline & $S P$ & 4122324 & 63,61 & 36,39 \\
\hline & Otra & 146483 & 36,55 & 63,45 \\
\hline & |MSS-Oportunidades & 40171 & 72,42 & 27,58 \\
\hline
\end{tabular}




\begin{tabular}{|c|c|c|c|c|}
\hline \multirow{4}{*}{$\begin{array}{c}\text { Trimestre de inicio de control } \\
\text { prenatal } \\
(n=8568292)\end{array}$} & No recibió & 207399 & 72,48 & 27,52 \\
\hline & Primero & 6439776 & 50,66 & 49,34 \\
\hline & Segundo & 1606077 & 63,12 & 36,88 \\
\hline & Tercero & 315040 & 63,04 & 36,96 \\
\hline \multirow{3}{*}{$\begin{array}{l}\text { Total de consultas prenatales } \\
\qquad(\mathrm{n}=\mathbf{8} 442990)\end{array}$} & 0 a 1 & 311315 & 70,17 & 29,83 \\
\hline & 2 a 5 & 1952815 & 64,44 & 35,56 \\
\hline & 6 o más & 6178860 & 49,94 & 50,06 \\
\hline \multirow{2}{*}{$\begin{array}{l}\text { Tipo de producto } \\
(\mathrm{n}=8692757)\end{array}$} & Único & 8549059 & 54,72 & 45,28 \\
\hline & Múltiple & 143698 & 14,2 & 85,8 \\
\hline \multirow{3}{*}{$\begin{array}{l}\text { Peso del recién nacido } \\
\qquad(n=8327896)\end{array}$} & Menos de 2,500 gramos & 549025 & 40,16 & 59,84 \\
\hline & $\begin{array}{l}\text { Entre 2,501 y 3,999 } \\
\text { gramos }\end{array}$ & 7498679 & 55,34 & 44,66 \\
\hline & 4,000 gramos o mas & 280192 & 46,57 & 53,43 \\
\hline \multirow{4}{*}{$\begin{array}{l}\text { Semanas de gestación } \\
\qquad(\mathrm{n}=8690862)\end{array}$} & Menos de 28 & 22716 & 60,68 & 39,32 \\
\hline & De 28 a 36 & 563444 & 35,36 & 64,64 \\
\hline & De 37 a 41 & 8002245 & 55,37 & 44,63 \\
\hline & De 42 a 45 & 102457 & 50,38 & 49,62 \\
\hline \multirow{7}{*}{$\begin{array}{l}\text { Sitio del parto } \\
(n=8566661)\end{array}$} & Secretaría de Salud & 4196145 & 66,04 & 33,96 \\
\hline & IMSS Oportunidades & 377525 & 73,11 & 26,89 \\
\hline & IMSS & 1796773 & 53,03 & 46,97 \\
\hline & ISSSTE & 153860 & 30,87 & 69,13 \\
\hline & $\begin{array}{l}\text { PEMEX, SEDENA O } \\
\text { SEMAR }\end{array}$ & 71380 & 50,84 & 49,16 \\
\hline & Otra unidad pública & 219340 & 52,07 & 47,93 \\
\hline & Unidad médica privada & 1751638 & 20,86 & 79,14 \\
\hline \multirow{3}{*}{$\begin{array}{l}\text { Orden de nacimiento } \\
\qquad(n=8662305)\end{array}$} & De 1 a 2 & 5782688 & 51,54 & 48,46 \\
\hline & De 3 a 5 & 2644469 & 57,62 & 42,38 \\
\hline & De 6 a 10 & 226967 & 73,88 & 26,12 \\
\hline
\end{tabular}




\begin{tabular}{|c|c|c|c|c|}
\hline & 11 o más & 8181 & 74,23 & 25,77 \\
\hline \multirow{5}{*}{$\begin{array}{l}\text { Días de estancia } \\
\text { (n = } 6569862)\end{array}$} & De 0 a 1 & 3070174 & 54,68 & 45,32 \\
\hline & De 2 a 7 & 1343374 & 54,94 & 45,06 \\
\hline & De 8 a 30 & 1113203 & 52,47 & 47,53 \\
\hline & De 31 a 99 & 610283 & 50,45 & 49,55 \\
\hline & 100 o más & 432828 & 52,27 & 47,73 \\
\hline \multirow{3}{*}{$\begin{array}{c}\text { Indígena } \\
(n=8673455)\end{array}$} & Con presencia indígena & 4528359 & 51,28 & 48,72 \\
\hline & Población indígena & 631310 & 66,72 & 33,28 \\
\hline & $\begin{array}{l}\text { Con población indígena } \\
\text { dispersa }\end{array}$ & 3511606 & 55,34 & 44,66 \\
\hline \multirow{4}{*}{$\begin{array}{l}\text { Índice de Desarrollo Humano } \\
\qquad(n=8673455)\end{array}$} & Bajo & 54377 & 79,82 & 20,18 \\
\hline & Medio bajo & 1391457 & 65,08 & 34,92 \\
\hline & Medio alto & 5744525 & 53,06 & 46,94 \\
\hline & Alto & 1483096 & 46,58 & 53,42 \\
\hline
\end{tabular}

Notas. IMSS Instituto Mexicano del Seguro Social, ISSSTE Instituto de Seguridad y Servicios Sociales para los Trabajadores del Estado, PEMEX Servicios de Salud de Petróleos Mexicanos, SEDENA Servicios de Salud de la Secretaría de la Defensa Nacional, SEMAR Servicios de Salud de la Secretaría de MarinaArmada de México, IMSS-Oportunidades Instituto Mexicano del Seguro Social - Oportunidades (actualmente Prospera)

Fuente: Elaboración de los autores con información de la Dirección General de Información en Salud (DGIS, 2015), Subsistema de Información de los Nacimientos Ocurridos (SINAC, 2011-2014) 
Tabla 2.

Regresión logística multivariada de factores asociados con la práctica de la cesárea en México, 2011-2014

\begin{tabular}{|c|c|c|c|c|}
\hline \multirow[t]{2}{*}{ Cesárea (1=sí, 2=no) } & \multirow{2}{*}{$\begin{array}{r}\text { Exp } \\
\text { (B) }\end{array}$} & \multirow[t]{2}{*}{$P>z$} & \multicolumn{2}{|c|}{$\begin{array}{c}\text { Intervalo de confianza al } \\
95 \%\end{array}$} \\
\hline & & & Inferior & Superior \\
\hline \multicolumn{5}{|l|}{ Estado conyugal de la madre } \\
\hline \multicolumn{5}{|l|}{ Alguna vez unida } \\
\hline Unida & 0,99 & 0,26 & 0,96 & 1,01 \\
\hline Soltera & 1,02 & 0,06 & 1,00 & 1,05 \\
\hline \multicolumn{5}{|l|}{ Escolaridad de la madre } \\
\hline \multicolumn{5}{|l|}{ Ninguna o primaria incompleta } \\
\hline Primaria completa & $1,08^{*}$ & 0 & 1,07 & 1,09 \\
\hline Secundaria completa & $1,15^{*}$ & 0 & 1,14 & 1,16 \\
\hline Bachillerato o preparatoria completa & $1,32^{*}$ & 0 & 1,31 & 1,33 \\
\hline Profesional o posgrado & $1,58^{*}$ & 0 & 1,57 & 1,60 \\
\hline \multicolumn{5}{|l|}{ Edad de la madre } \\
\hline \multicolumn{5}{|l|}{ Menos de 17} \\
\hline $18-24$ & $1,03^{*}$ & 0 & 1,02 & 1,03 \\
\hline 25 a 34 & $1,36^{*}$ & 0 & 1,35 & 1,37 \\
\hline 35 a 44 & $2,01^{*}$ & 0 & 1,99 & 2,03 \\
\hline 45 o más & $2,35^{*}$ & 0 & 2,24 & 2,47 \\
\hline \multicolumn{5}{|l|}{ Derechohabiencia de la madre } \\
\hline \multicolumn{5}{|l|}{ Ninguna } \\
\hline IMSS & $1,21^{*}$ & 0 & 1,19 & 1,22 \\
\hline ISSSTE & $1,27^{*}$ & 0 & 1,24 & 1,31 \\
\hline PEMEX, SEDENA O SEMAR & $1,23^{*}$ & 0 & 1,16 & 1,31 \\
\hline Seguro Popular & $0,98^{*}$ & 0 & 0,98 & 0,99 \\
\hline Otra & $1,06^{*}$ & 0 & 1,04 & 1,08 \\
\hline IMSS Oportunidades & 1,02 & 0,12 & 0,99 & 1,06 \\
\hline \multicolumn{5}{|l|}{ Trimestre de inicio de control prenatal } \\
\hline \multicolumn{5}{|l|}{ No recibió } \\
\hline Primero & $1,09^{*}$ & 0 & 1,07 & 1,12 \\
\hline Segundo & 0,99 & 0,57 & 0,97 & 1,02 \\
\hline Tercero & $0,97 *$ & 0,02 & 0,95 & 0,99 \\
\hline
\end{tabular}




\section{Total de consultas prenatales}

De 0 a 1

de 2 a 5

6 o más

\section{Parto múltiple}

No

Sí

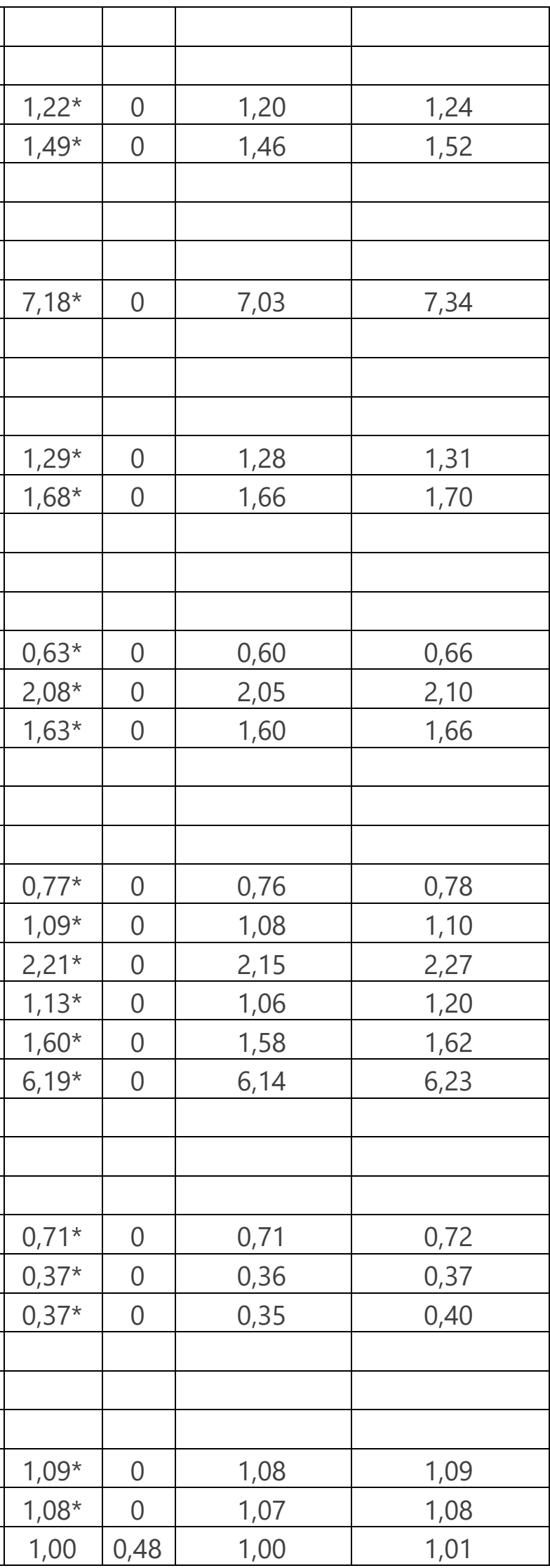

Peso del recién nacido

De 2500 a 3999

menos de 2500

4000 o más

\section{Semanas de gestación}

De 37 a 41

menos de 28

de 28 a 36

de 42 a 45

\section{Sitio del parto}

Secretaría de Salud

IMSS Oportunidades

IMSS

ISSSTE

PEMEX, SEDENA o SEMAR

Otra unidad pública

Unidad médica privada

\section{Orden de nacimiento}

De 1 a 2

De 3 a 5

De 6 a 10

11 o más

\section{Días de estancia}

De 0 a 1

2 a 7

8 a 30

31 a 99 


\begin{tabular}{|l|c|c|c|c|}
\hline 100 o más & $1,04^{*}$ & 0 & 1,03 & 1,04 \\
\hline Presencia indígena & & & & \\
\hline Con presencia indígena & & & & \\
\hline Población indígena & & & & \\
\hline Con población indígena dispersa & $1,12^{*}$ & 0 & 1,11 & 1,14 \\
\hline & $1,06^{*}$ & 0 & 1,05 & 1,06 \\
\hline Índice de Desarrollo Humano & & & & \\
\hline Bajo & & & & \\
\hline Medio bajo & & & & \\
\hline Medio alto & $1,34^{*}$ & 0 & 1,30 & 1,39 \\
\hline Alto & $1,68^{*}$ & 0 & 1,63 & 1,74 \\
\hline
\end{tabular}

* La estimación es significativa al 0.05

Notas. IMSS Instituto Mexicano del Seguro Social, ISSSTE Instituto de Seguridad y Servicios Sociales para los Trabajadores del Estado, PEMEX Servicios de Salud de Petróleos Mexicanos, SEDENA Servicios de Salud de la Secretaría de la Defensa Nacional, SEMAR Servicios de Salud de la Secretaría de Marina-Armada de México, IMSS-Oportunidades Instituto Mexicano del Seguro Social - Oportunidades (actualmente Prospera)

Fuente: Elaboración de los autores con información de la Dirección General de Información en Salud (DGIS, 2015), Subsistema de Información de los Nacimientos Ocurridos (SINAC, 2011-2014) 


\section{Población y en Mesoamérica}

¿Quiere publicar en la revista?

Ingrese aquí

O escríbanos:

revista@ccb.ucr.ac.c

\section{$1^{\circ}$ Revista}

Electrónica

13 años de liderazgo

Enlace Award, 2007

\section{UCR Index}

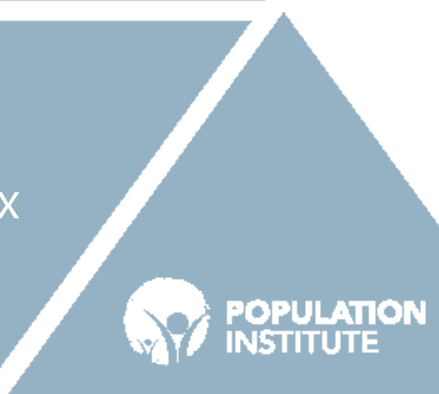

Global Media Awards

Excellence in Population Reporting

Población y Salud en Mesoamérica (PSM) es la revista electrónica que cambió el paradigma en el área de las publicaciones científicas electrónicas de la UCR. Logros tales como haber sido la primera en obtener sello editorial como revista electrónica la posicionan como una de las más visionarias.

\section{Revista PSM es la letra delta mayúscula, el cambio y el futuro.}

Indexada en los catálogos más prestigiosos. Para conocer la lista completa de índices, ingrese aquí.

sciglo DOAJ Iatindex "redalyc.org o Dialnet e-revist@s 0 clarivate

Revista Población y Salud en Mesoamérica -

Centro Centroamericano de Población

Universidad de Costa Rica

\section{(6) (2)}

